\title{
A framework for an evidence-based gene list relevant to autism spectrum disorder
}

Christian P. Schaaf ${ }^{1,2,3}$, Catalina Betancur ${ }^{4}$, Ryan K. C. Yuen ${ }^{5,6}$, Jeremy R. Parr ${ }^{7}$, David H. Skuse $^{8}$, Louise Gallagher 9 , Raphael A. Bernier ${ }^{10,11}$, Janet A. Buchanan, Joseph D. Buxbaum $^{12,13}$, Chun-An Chen ${ }^{2}$, Kira A. Dies ${ }^{14}$, Mayada Elsabbagh ${ }^{15}$, Helen V. Firth ${ }^{16}$, Thomas Frazier $^{17}$, Ny Hoang ${ }^{5}$, Jennifer Howe ${ }^{5}$, Christian R. Marshall ${ }^{5}$, Jacques L. Michaud ${ }^{18}$, Olivia Rennie $^{5}$, Peter Szatmari ${ }^{19,20}$, Wendy K. Chung ${ }^{21}$, Patrick F. Bolton ${ }^{22}$, Edwin H. Cook ${ }^{23}$, Stephen W. Scherer ${ }^{5,6,24} *$ and Jacob A. S. Vorstman ${ }^{5,19,25 *}$

${ }^{1}$ Institute of Human Genetics, Heidelberg University, Heidelberg, Germany.

${ }^{2}$ Department of Molecular and Human Genetics, Baylor College of Medicine, Houston, TX, USA.

3Jan and Dan Duncan Neurological Research Institute, Texas Children's Hospital, Houston, TX, USA.

${ }^{4}$ Neuroscience Paris Seine, Institut de Biologie Paris Seine, Sorbonne Université, INSERM, CNRS, Paris, France.

${ }^{5}$ The Centre for Applied Genomics, Program in Genetics and Genome Biology, The Hospital for Sick Children, Toronto, ON, Canada.

${ }^{6}$ Department of Molecular Genetics, University of Toronto, Toronto, ON, Canada.

${ }^{7}$ Population Health Science Institute, Sir James Spence Institute, Newcastle University, Royal Victoria Infirmary, Newcastle, UK.

${ }^{8}$ Institute of Child Health, University College London, London, UK.

${ }^{9}$ Department of Psychiatry \& Neuropsychiatric Genetics Research Group, School of Medicine, The Trinity Centre for Health sciences, Trinity College Dublin, Dublin, Ireland.

${ }^{10}$ Department of Psychiatry and Behavioral sciences, University of Washington, Center for Child Health, Behavior, and Disabilities, Seattle, WA, USA.

${ }^{11}$ Children's Autism Center, Seattle, WA, USA.

${ }^{12}$ Department of Psychiatry, Icahn School of Medicine at Mount sinai, New York, NY, USA.

${ }^{13}$ Department of Genetics and Genomic sciences, Icahn School of Medicine at Mount sinai, New York, NY, USA.

${ }^{14}$ Translational Neuroscience Center, Department of Neurology, Boston Children's Hospital, Harvard Medical School, Boston, MA, USA.

${ }^{15}$ Montreal Neurological Institute, Azrieli Centre for Autism Research, McGill University, Montreal, QC, Canada.

${ }^{16}$ Department of Clinical Genetics, Cambridge University Hospitals NHS Foundation Trust \& Wellcome Sanger Institute, Wellcome Genome Campus, Hinxton, Cambridge, UK.

${ }^{17}$ Autism Speaks, Cleveland, OH, USA.

${ }^{18} \mathrm{CHU}$ Sainte-Justine Research Center, University of Montreal, Montreal, QC, Canada.

${ }^{19}$ Department of Psychiatry, Hospital for Sick Children, Toronto, ON, Canada.

${ }^{20}$ Centre for Addiction and Mental Health, University of Toronto, Toronto, ON, Canada.

${ }^{21}$ Department of Pediatrics (in Medicine), Columbia University Medical Center, New York, NY, USA.

${ }^{22}$ Institute of Psychiatry, King's College London, London, UK.

${ }^{23}$ Department of Psychiatry, University of Illinois at Chicago, Chicago, IL, USA.

${ }^{24}$ McLaughlin Centre, University of Toronto, Toronto, ON, Canada.

${ }^{25}$ Department of Psychiatry, University of Toronto, Toronto, ON, Canada.

*e-mail: stephen.scherer@sickkids.ca, jacob.vorstman@sickkids.ca 


\section{Abstract}

Autism spectrum disorder (ASD) is often grouped with other brain-related phenotypes into a broader category of neurodevelopmental disorders (NDDs). In clinical practice, providers need to decide which genes to test in individuals with ASD phenotypes, which requires an understanding of the level of evidence for individual NDD genes that supports an association with ASD. Consensus is currently lacking about which NDD genes have sufficient evidence to support a relationship to ASD. Estimates of the number of genes relevant to ASD differ greatly among research groups and clinical sequencing panels, varying from a few to several hundred. This Roadmap discusses important considerations necessary to provide an evidence-based framework for the curation of NDD genes based on the level of information supporting a clinically relevant relationship between a given gene and ASD.

\section{Introduction}

Autism spectrum disorder (ASD) is a multifactorial neurodevelopmental disorder (NDD) characterized by impairments in social communication and repetitive and restricted behaviours and interests. While this disorder is defined behaviourally rather than genetically or biologically ${ }^{1}$, at present a contributing genetic variant can be identified in $5-30 \%$ of individuals with ASD, depending on the genetic test used, the cohort examined and the thresholds used for significance ${ }^{2-6}$. Genetic variants ranging from extremely rare to common, including de novo and inherited variants, affect genes in a manner that together or on their own can result in an ASD phenotype ${ }^{4,7}$. Current clinical guidelines therefore recommend genetic testing of individuals (usually children) with a diagnosis of ASD to determine any underlying genetic susceptibility that contributes to the disorder ${ }^{8}$. Although identifying the genetic contribution to the ASD phenotype cannot replace the behavioural diagnosis, it may help to explain its aetiology, inform the likelihood of recurrence, which can be important for family planning, support management of associated medical conditions, and potentially support genetically tailored treatment in the future ${ }^{9-11}$. This approach is in line with the revised classification of ASD in the Diagnostic and Statistical Manual of Mental Disorders (DSM-5) ${ }^{12}$, whereby "association with a known genetic condition" can be annotated as a specification ${ }^{13}$. While the specifier confirms a defined genetic contribution to the observed ASD phenotype, it does not necessarily imply a specific behavioural subtype of ASD, and the diagnosis of ASD remains behaviourally defined.

Clinical genetic testing for ASD has increased and will continue to expand with the wider implementation of high-throughput sequencing ${ }^{14}$. Having a list of genes with sufficient evidence to support a relationship with ASD is crucial for the genome-wide testing of individuals with ASD (Box 1). The availability of a list of genes systematically curated for ASD relevance would increase the consistency of testing across laboratories and decrease the burden of curation, as many genes for ASD are likely still to be identified. An ASD-relevant gene list will also be useful when sequencing individuals with ASD, to focus the search for mutations and increase the efficiency of analysis.

To address the complexity inherent to ASD genetics, the field continues to develop statistical methods to identify common variants with individually small effect sizes through genome-wide association studies as well as rare variants identified in sequencing studies that approximate a Mendelian pattern of disease inheritance ${ }^{15-17}$. Although important to advance the field, these 
methods do not provide an unequivocal strategy to evaluate the extent to which available evidence supports a clinically relevant relationship between a given gene and ASD. As a result, there is substantial variability in the genes included on autism genetic testing panels and the lists of genes used to interrogate the exome or genome for an ASD phenotype ${ }^{18}$.

The lack of a standardized approach for evaluating evidence supporting a clinically relevant relationship between a gene and ASD represents a challenge in clinical practice, both for providers ordering genetic tests and the laboratories offering the respective panels ${ }^{18}$. ASD is often grouped with other brain-related phenotypes, such as intellectual disability (ID) and epilepsy, into a broader category of NDDs. To the best of our knowledge, all genes implicated in ASD to date are also involved in at least one other NDD. Currently, there is no consensus about which NDD genes have sufficient evidence to support a relationship to ASD.

Guidance was sought from an international multi-disciplinary group of experts in ASD and clinical genetics, who have met remotely for the past year following a 2-day meeting in September 2018, sponsored by the University of Toronto McLaughlin Centre. The group reviewed existing gene-disease validity evaluation frameworks ${ }^{19-22}$ to determine whether one could be adopted to serve the needs of the clinical community for documentation of genes relevant to ASD. As the purpose is to define an ASD-relevant gene list for use in a clinical setting, criteria for inclusion will be more stringent than they would be for a discovery-driven agenda ${ }^{23,24}$. The Clinical Genome (ClinGen) Gene-Disease Validity curation framework was selected as a starting point. ClinGen is an initiative that has developed a systematic framework explicitly aimed at assessing the clinical relevance of genes, and this framework has been implemented successfully for an increasing number of phenotypes, including NDDs ${ }^{19,21,22}$. In this Roadmap, we propose that standardized and transparent evaluation of the evidence supporting the relationship of a gene to the ASD phenotype is an important next step. Ideally, the outcome of such additional evaluation would be embedded as an additional annotation to each of the genes curated for NDD through such a framework.

\section{Neurodevelopmental disorders}

The term NDD is widely used in research and clinical practice and describes a range of developmental disorders associated with the central nervous system, including but not limited to ID, ASD and epilepsy. However, NDD is neither a clinically valid diagnosis nor a diagnostic classification term. Of note, there is no uniformly accepted definition of NDD; for instance, in the DSM- ${ }^{12}$, NDD encompasses a broad range of over 25 disorders, including ID, ASD, attention deficit hyperactivity disorder (ADHD), communication disorders, specific learning disorders, stuttering and tic disorders ${ }^{12}$. In a research context, the definitions of NDD are variable and may or may not include epilepsy, schizophrenia, bipolar disorder and specific genetic disorders ${ }^{25-28}$.

Despite these variable definitions, the NDD grouping provides a useful concept, as it captures the full breadth of phenotypic impact of many pathogenic variants. The NDD concept is widely adopted in laboratory testing and clinical genetics ${ }^{29,30}$. From a pragmatic perspective, this is a reasonable starting strategy; once genes with strong evidence for a role in NDDs are identified, the 
more specific relationship to any subsumed phenotypes, such as ASD, epilepsy or ID, can be evaluated. From an epidemiological perspective, this first step is further understandable given the high co-morbidity among these disorders, which exceeds that which would be expected by chance $^{1}$.

Of note, while genes thus far associated with ASD have also been associated with other brainrelated disorders commonly under the umbrella term NDD - in particular ID and epilepsy ${ }^{31}-$ genes may yet be identified that are more relevant to ASD and less so (or not at all) to other NDDs. Indeed, recent studies provide evidence for variants that significantly affect ASD phenotypes and less so intelligence quotient ${ }^{4,32-34}$. However, in the context of current evidence, when evaluating gene-phenotype validity, consideration of the compound NDD phenotype is rational ${ }^{28,30}$. At the same time, knowledge about a gene association that is specific to the ASD phenotype may be valuable in clinical care for the individual patient and family, and useful to focus research questions in precision medicine concerning $\mathrm{ASD}^{11,25}$. In this regard, the relationship between ASD and ID may be of particular interest.

ASD and intellectual disability. Epidemiological studies show that ASD and ID can manifest independently. About $55 \%$ of ASD diagnoses involve individuals in the average to (very) high range of intellectual functioning ${ }^{1}$, whereas approximately $60-90 \%$ of individuals with ID (including profound ID) do not meet criteria for $\mathrm{ASD}^{35}$. Current insights into the complex relationship between these two phenotypes are evolving ${ }^{36}$ as illustrated, for example, by the observation that ASD prevalence in the USA has increased simultaneously with a decrease in reported population rates of $1 \mathrm{D}^{37-39}$. Of note, many large-scale studies analyse genetic variants for aggregate phenotypes, preventing identification of phenotype-specific effect of genes that may exist.

ASD and ID have different attributes: ASD is characterized by atypical social communication, the presence of restricted or repetitive behaviours or strong interests, and preoccupations that interfere with function ${ }^{1}$. By contrast, ID comprises impaired intellectual and adaptive functioning. The nosological distinction between ASD and ID is well supported, indicating that behavioural symptoms reliably discriminate between the two ${ }^{40,41}$ and distinguish concurrent ID and ASD ${ }^{42,43}$. Of note, several behaviours - including responsiveness to name ${ }^{44,45}$, emotional expression ${ }^{45}$ and eye gaze pertinent to social interactions $s^{44,46}$ - can sometimes distinguish between these disorders as early as 12 months of age ${ }^{47}$.

Recognizing ASD as a phenotype when assessing the clinical relevance of a gene is important because of existing differences in the clinical management of people with only ASD, people with ASD and ID, and those with only ID. Features such as developmental expectations, areas of adaptive strengths and weaknesses, patterns of co-morbid psychopathology ${ }^{48}$, academic needs and required support ${ }^{49}$ are all relevant to prognosis and treatment choices ${ }^{50}$.

ASD and epilepsy. Like ASD, epilepsy can be considered one of the NDD phenotypes. Similar to ASD, epilepsy is a heterogeneous condition, with many genes and co-morbidities involved. Epilepsy, therefore, stands to benefit from the evaluation of evidence in support of a gene's relationship to a 
seizure disorder specifically, because it is a distinct phenotype, with clinical implications that differ from other NDD phenotypes. Indeed, application of the ClinGen framework for the curation of genes relevant to epilepsy has recently been initiated ${ }^{51}$.

\section{Heterogeneity in the evaluation of ASD}

An effective curation process depends on high-quality data from both the genotype and phenotype arms ${ }^{21,22}$. For a behaviourally defined condition such as ASD, where the key features can overlap with several other NDDs, the definition of the phenotype being curated is crucial. A diagnosis of ASD should be made according to conventional diagnostic criteria, as defined by the DSM $-5^{12}$ and the International Statistical Classification of Diseases and Related Health Problems 10th Revision (ICD-10) ${ }^{52}$, based on a phenotypic description of difficulties in social communication and restricted, repetitive behaviours ${ }^{1}$. The diagnostic process typically involves direct observation and an evaluation of developmental history from multiple informants (for example, parents, teachers and family physicians) preferably by a trained clinician and supported by the use of standardized assessment tools ${ }^{1}$. It is well recognized that there is variability in the diagnostic approach used by different clinicians (for example, neurologist, developmental pediatrician, child psychiatrist or psychologist) and in clinical versus research settings. As a result, the quality of the diagnostic information reported can vary, which may influence diagnosis in cohorts being collected for genetic studies. In addition, descriptions of the phenotype and phenotyping process for ASD are highly variable in the published literature. Some studies explicitly mention conventional (DSM/ICD) criteria, often with documentation of the ASD assessment tools or diagnostic process used, whereas others only mention 'ASD' or even 'ASD traits' or 'autistic behaviour'. This variability in the quality of reporting ASD phenotypes is a major concern in the process of evaluating genetic associations and should be taken into account when interpreting evidence.

\section{Genetic complexity of ASD}

The complex genetic architecture of ASD makes it difficult to delineate the specific clinical impact of gene variants. By studying ASD cohorts, an increasing number of genes are being identified that, when having altered function or dosage, can result in or include an ASD phenotype in a proportion of carriers ${ }^{4,34,53,54}$. Examples include copy number variation (CNVs), such as deletions or duplications at 16p11.2 (ASD expressed in 20-25\% carriers) ${ }^{55,56}$, deletions at 22q11.2 (ASD expressed in $\sim 20-25 \%)^{57,58}$ or $22 q 13.3$ (ASD expressed in $\left.84 \%\right)^{59}$, as well as sequence variants (usually rare, de novo or inherited variants) in genes such as ARID1B, CHD8 or SCN2A ${ }^{33,60-62}$. Of note, for some genes affected by deleterious variants, the associated prevalence of ASD is higher than that of ID, indicating that at least a proportion of individuals has ASD without ID $^{32-34,63}$ (for example, some individuals affected by pathogenic variants in $P T E N^{64}$ ). However, the evaluation of the penetrance of ID and ASD is likely to be affected by the imposition of a categorical division onto a quantitative phenotype. Furthermore, the impact of a pathogenic variant could be obscured when the phenotype falls within population norms, despite deviation from the expected given the phenotype in parents and siblings without the pathogenic variant ${ }^{29}$. However, the 
categorical distinction (for example, between those with and those without ID or ASD) remains valuable and constitutes much of the basis on which gene-phenotype relationships are currently assessed. From this viewpoint, it is noteworthy that reports on individuals with pathogenic variants include probands with ASD who do not have ID (for example, $C H D 8^{62}$, duplications or deletions at $16 \mathrm{p} 11.2^{55,65,66}$, and deletions at the X-linked PTCHD1-AS locus ${ }^{67}$ ).

Adding to the genetic complexity of ASD $^{68}$ are the roles of somatic mosaicism ${ }^{69}$, differences in phenotypic expression of ASD between male and female individuals ${ }^{70}$, pleiotropy, reduced penetrance and variable expressivity - phenomena that may also have an impact on clinical phenotypic manifestations. Pleiotropy refers to the impact of the same genetic variant in multiple systems or tissues (for example, cardiac defects and endocrine disorders in addition to ASD $^{71,72}$ ). Reduced penetrance means that the genetic variant does not always manifest with a phenotype. Variable expressivity refers to the range of manifestations of a given genotype among different individuals with the same genetic variant (for example, levels of ID severity) ${ }^{73}$. These phenomena are not specific to ASD but may also be important in other disorders or phenotypes grouped under NDDs, including $I D^{29}$. The incomplete penetrance and variable expressivity of ASD in the presence of a high-impact genetic variant may be under the influence of additional genetic variation ${ }^{74-76}$, including common genetic variation ${ }^{7}$ or epigenetic factors ${ }^{77-79}$ and possibly environmental contributions $^{80}$. A polygenic risk score, which expresses the cumulative impact of thousands of common variants on the probability of a phenotypic outcome, for ASD explains approximately $2.5 \%$ of the observed variance ${ }^{81}$ and has therefore no clinical use as a risk prediction tool in the general population at this time. With such genetic and phenotypic complexity underlying ASD, there needs to be a consensus framework to assess the relevance of NDD genes to ASD. This issue is all the more relevant because some genetic studies combine cases of ASD and other non-ASD phenotypes within the NDD group, apparently to increase sample size (for example, see Refs. ${ }^{82,83}$ ), which can influence results, including the composition of gene lists arising from these studies.

\section{A gene evaluation framework for ASD}

Strategies have been proposed to systematically assess the evidence for involvement of genes in disease aetiology ${ }^{20,84}$. For the ASD research community, the SFARI Gene database [https://gene.sfari.org/] is a well-known resource in this regard ${ }^{85}$, and guided by a panel of advisors, it provides a growing list of genes ( $n=913$ at present, grouped into tiers), for which publication evidence of association with ASD is presented. However, although SFARI Gene is a structured and valuable resource ${ }^{84}$, it currently does not seem to provide a systematic curation framework for evaluating the extent to which genes have a clinically relevant relationship with ASD, perhaps favouring research questions. A recently published clinical report on the evaluation and treatment of ASD by the American Academy of Pediatrics (AAP) ${ }^{86}$ lists 22 selected ASD risk genes identified or confirmed in whole-exome studies, which includes $K D M 5 B$, a gene that does not achieve the highest SFARI score for association with ASD risk. A recent review on ASD genetics ${ }^{87}$ and a recent genome sequencing study ${ }^{33}$ further illustrate the current lack of consensus (Fig. 1). 
The ClinGen curation process. The ClinGen Gene-Disease Validity curation process evaluates the level of evidence supporting a given gene-phenotype relationship and stratifies this into six classifications (from refuted to definitive) using both genetic and experimental evidence. The curation process has been outlined previously ${ }^{19}$ and follows a detailed Standard Operating Procedure (Ref-Version 6, August 29, 2018). Briefly, the following are critical steps in the procedure. First, the disease entity for which the gene will be curated is established, as is the mode of inheritance. For the purposes of the proposed framework, the disease entity is defined as ASD, regardless of the presence or absence of co-morbid phenotypes. The second step consists of the (broad) collection of evidence including peer-reviewed literature and variant databases. Genetic evidence is assessed based on various study types, including case reports and case series, but also statistical associations reported in case-control and linkage studies. Experimental evidence is evaluated, including expression studies, in vitro, ex vivo and in vivo studies, always taking construct validity and face validity into consideration. Acknowledging that interpretation of experimental evidence is often not indisputable, in particular with regard to neurodevelopmental and psychiatric conditions, the curation system limits the number of points that can be achieved in the experimental category. Thus, the final classification cannot get beyond the lowest category ('Limited') unless genetic evidence is also available. Third, all evidence is summarized and scored using a detailed scoring matrix. The fourth and final step consists of a multidisciplinary expert review of evidence, which generates the final classification for the gene-disease relationship. This ClinGen framework was originally intended for a variety of Mendelian disorders and has been incrementally adapted for more complex disorders ${ }^{22}$.

Adapting the ClinGen framework for ASD. Building on the existing ClinGen curation efforts towards a list of NDD-associated genes (which sometimes draw from studies of ASD), we propose the same framework be applied to evaluate the evidence for involvement of NDD genes in ASD through a curation process that weighs the quality of peer-reviewed evidence (Fig. 2). Genes for curation are selected based on their reported relevance to ASD (beginning with those from existing NDD lists), starting with strong candidates, as judged by the number of independent peer-reviewed studies and individuals reported with relevant variants. To address the variable quality of information available regarding the ASD phenotype, we propose an algorithm for its systematic evaluation (Fig. 3), the output of which is used in the gene curation procedure. Scoring adjustments will account not only for quality of the information available on the ASD phenotype (Fig. 3) but also issues specific to ASD (Table 1). For example, additional aspects, currently not yet taken into consideration in the curation process, include the higher phenotypic resilience or different phenotypic expression in women ${ }^{88-90}$, as well as the fact that parents or siblings may have ASD even in the absence of the putatively pathogenic variant ${ }^{32,91,92}$. After attributing an evidence-based score for a gene's relevance to ASD, additional comments may be added (pleiotropic effects, incomplete penetrance and variable expressivity). This information will be made publicly available and updated on an ongoing basis. 
The principal question to be addressed by the curation process is whether a given gene, when affected by a deleterious variant, could account for phenotypic findings of ASD in the individual carrying that genetic variant, regardless of any other associated phenotype (for example, ID or epilepsy). The outcome of the curation process reflects the results of a systematic evaluation of all available evidence regarding a possible relationship between a gene and ASD specifically. Importantly, specificity does not imply exclusivity; the same gene can also be associated with other phenotypic outcomes.

\section{Exemplary use of the proposed strategy}

To demonstrate the proposed approach, we curated eleven potential ASD genes: ADNP, ANK2, ARID1B, DSCAM, KATNAL2, KDM5B, MEF2C, NLGN4X, NRXN2, SYN1 and VPS13B. These genes illustrate how the proposed framework for ASD specifically may generate classifications that differ as a function of source (for example, ClinGen, SFARI, AAP Clinical Report publication). For each gene, we scrutinized all publications indicating a relationship between the gene and ASD, extracting from main text and supplementary materials all available details about the diagnostic methods used and descriptions of ASD phenotype and intellectual ability. First, for each case contributing to genetic evidence we generated a preliminary score following the ClinGen framework. Subsequently, we determined the level of confidence in the reported ASD phenotype for each counted case, based on the systematic evaluation of the quality of diagnostic method and information on cognitive ability (Fig. 3). The level of confidence in the reported ASD phenotype was then used to adjust the preliminary ClinGen framework scores into a final score that was tallied and categorized to reflect the level of evidence available to support a relationship between the given gene and ASD (Table 2). Supplementary Table 1 provides detailed information on the scoring process. Variants with similar predicted impact on protein function were awarded fewer points when confidence in the reported ASD phenotype was low, compared to cases with a high-confidence phenotype. This process is illustrated in the scoring of VPS13B. Frameshift variants reported in Family AU-21100 explicitly mentioned that an experienced clinician (neurologist, child psychiatrist or psychologist) provided the ASD diagnosis ${ }^{93}$. By contrast, another study also reported frameshift variants, but without information about how the ASD diagnosis was derived ${ }^{94}$. To reflect the lower confidence in the ASD phenotype in the latter study, one point was deducted from the default ( 2 points), whereas no points were deducted from the first case (Supplementary Table 1).

Cognitive ability information can lower the confidence in the ASD phenotype despite the use of gold standard testing methods. For instance, in the scoring of $N L G N 4 X$, a male patient was diagnosed as having ASD according to DSM-IV criteria, but profound ID was also noted ${ }^{95}$. In individuals with profound ID, the validity of the ASD diagnosis is reduced ${ }^{96}$; hence, scoring for this case was adapted to reflect that. The ClinGen procedure recommends score adjustment if variants similar to those observed in probands are also observed in unaffected individuals. This may explain why some genes generated lower classifications by our curation even though these genes are considered robust ASD risk genes in the existing literature. For example, both KATNAL2 and ANK2 received the highest score by SFARI Gene and are included in the 22 selected ASD risk genes by the American Academy of 
Pediatrics $^{86}$. For KATNAL2, the $\mathrm{pLI}$ score listed in the gnomAD database (https://gnomad.broadinstitute.org/) is 0 , indicating that loss-of-function variants are tolerated in the population. Indeed, loss of function variants reported in this gene are frequently also observed in clinically unaffected parents ${ }^{32,60}$, which puts into question whether haploinsufficiency of KATNAL2 is a plausible mechanism associated with ASD risk. Along the same line of reasoning, ANK2 scoring was downgraded because the missense variants observed in this gene were reported also in unaffected individuals ${ }^{97,98}$, and evidence of an impact on protein function was lacking.

Additional scoring adjustments can be considered to reflect the genetic complexity in ASD. For example, in contrast to the evaluation of typical Mendelian disorders, in ASD, an autosomal dominant variant transmitted from an unaffected mother to an affected son may be considered contributory ${ }^{99}$. Similarly, a variant not shared between two affected siblings may still be relevant ${ }^{32,92}$ (Supplementary Table 1).

\section{Reporting gene associations with ASD}

The proposed systematic evaluation of existing genetic studies in ASD can be used as a guideline for investigators planning to report on genetic findings in patients with ASD (Box 2). Some of the challenges related to the quality of the ASD phenotype are the consequence of missing information that may have been avoidable. For example, not reporting the assessment methods that were used to diagnose ASD reduces the degree of confidence in the phenotype. Similarly, it is essential to report cognitive assessment and results. The term "(global) developmental delay" seems to be used frequently to suggest a certain degree of ID, whereas it is actually a non-specific term for young children who are not meeting or have not met developmental milestones ${ }^{12}$. Unfortunately, in case reports it is often used as a phenotypic descriptor in older individuals, which makes it difficult to interpret; theoretically, the subject may have anywhere from profound ID to intellectual abilities within the normal IQ range. Similarly, the interpretability of genotype findings can be complicated by the use of ambiguous nomenclature, or lack of reporting of technologies, including aspects of the informatic pipelines such as the version of the genome-build used.

\section{Conclusions}

For queries about genetic testing in ASD, there is a need to robustly document the level of evidence in support of the involvement of an NDD-associated gene with ASD itself. The subset of genes that, when altered, result in a high likelihood of an ASD phenotypic outcome is fairly straightforward to evaluate. Those genes with lower relative risk of ASD require the development of thresholds to define the contribution to ASD probability and a system to quantify the proportion of ASD among non-ASD phenotypes under the NDD umbrella. At present, for genes included in existing diagnostic panels, it is often unclear whether and how these parameters were assessed, or to what extent genes were included based on association predominantly with other non-ASD neurodevelopmental phenotypes.

With a curated ASD-relevant gene list, clinical laboratories can design and/or test panels and/or gene prioritization algorithms based on the currently available evidence, and health care providers 
can order and interpret genomic tests with more confidence, according to their needs. Test indications may be set broadly for NDDs that are unspecified at the time of genetic testing, using the complete NDD gene list, or offer to focus on the most relevant genes for other disorders, such as epilepsy. The ClinGen framework provides a good path forward to at least initially examine rare, presumed high-impact variants in NDD genes for their role more specifically in ASD. Of note, other psychiatric conditions, such as schizophrenia, may benefit from a similar curation framework outlined in this manuscript.

We believe it is imperative that a broad, international consensus be reached regarding genes related to the aetiology of ASD and the strength of the evidence supporting each such gene. We currently fail to exploit the full potential of published genetic studies in ASD, in part because the phenotypic and genotypic information in a substantial number of reports is incomplete and/or ambiguous. Thus, we propose a systematic evaluation framework model that can be used to build an evidence-based list of genes relevant to ASD. The elements composing this framework also provide guidelines for researchers for future publications, maximizing their contribution to our understanding of the genetic underpinnings of ASD.

\section{Acknowledgements}

Christian Schaaf is supported by CDMRP Grant AR160154, the Foundation for Prader-Willi Research, the NR2F1 Foundation, and the USP7 Foundation. Dies Kira is supported by Boston Children's Hospital Neuroscience Clinical Cluster grant. Edwin Cook is supported by National Institute of Mental Health grant R01MH110920. Peter Szatmari is supported by Canadian Institutes of Health Research (CIHR) funding. Stephen Scherer is supported by GlaxoSmithKline-ClHR Endowed Chair in Genome Sciences at the Hospital for Sick Children and University of Toronto. Jacob Vorstman is funded by National Institute of Mental Health grant 1U01MH11974101 and $\mathrm{CIHR}$.

\section{Author contributions}

C.P.S., C.B., N.H., J.H., O.R. and J.A.S.V. researched the literature. C.P.S., C.B., R.K.C.Y., J.R.P., D.H.S., L.G., R.A.B., J.A.B., J.D.B., C.-A.C., K.A.D., M.E., H.V.F., T.F., N.H., J.H., C.M., J.L.M., P.S., W.K.C., P.F.B., E.H.C., S.W.S. and J.A.S.V. provided substantial contributions to discussions of the content. C.P.S., C.B., N.H., S.W.S. and J.A.S.V. wrote the article. C.P.S., C.B., R.K.C.Y., J.R.P., D.H.S., L.G., R.A.B., J.A.B., J.D.B., K.A.D., M.E., H.V.F., T.F., N.H., J.H., C.M., J.L.M., P.S., W.K.C., P.F.B., E.H.C., S.W.S. and J.A.S.V. reviewed and/or edited the manuscript before submission.

\section{Competing interests}

H.V.F. is a section editor for Genetics for UpToDate. W.K.C. declares that she is part of the Regeneron Genetics Center Scientific Advisory Board. S.W.S. declares he is part of scientific advisory committees and/or has intellectual property licensed to Deep Genomics, Population Bio, Lineagen, Athena Diagnostics.

\section{References}

1. Lai, M.C., Lombardo, M.V. \& Baron-Cohen, S. Autism. Lancet 383, 896-910 (2014).

2. Tammimies, K. et al. Molecular Diagnostic Yield of Chromosomal Microarray Analysis and Whole-Exome Sequencing in Children With Autism Spectrum Disorder. Jama-Journal of the American Medical Association 314, 895-903 (2015).

This paper shows among a heterogeneous sample of children with ASD that the diagnostic yield was higher in children with more complex morphological phenotypes.

3. Feliciano, P. et al. Exome sequencing of 457 autism families recruited online provides evidence for autism risk genes. NPJ Genom Med 4, 19 (2019). 
4. Sanders, S.J. et al. Insights into Autism Spectrum Disorder Genomic Architecture and Biology from 71 Risk Loci. Neuron 87, 1215-33 (2015).

This large study uses microarray and sequencing data to reveal strong evidence that de novo mutations are associated with ASD apart from the risk for ID.

5. Ronemus, M., lossifov, I., Levy, D. \& Wigler, M. The role of de novo mutations in the genetics of autism spectrum disorders. Nat Rev Genet 15, 133-41 (2014).

6. Shen, Y. et al. Clinical genetic testing for patients with autism spectrum disorders. Pediatrics 125, e727e735 (2010).

7. Weiner, D.J. et al. Polygenic transmission disequilibrium confirms that common and rare variation act additively to create risk for autism spectrum disorders. Nat Genet 49, 978-985 (2017).

This important study demonstrates that polygenic variation contributes additively to risk in individuals with ASD who carry a presumed penetrant de novo variant.

8. Barton, K.S. et al. Pathways from autism spectrum disorder diagnosis to genetic testing. Genet Med 20, 737-744 (2018).

9. Hoang, N., Cytrynbaum, C. \& Scherer, S.W. Communicating complex genomic information: A counselling approach derived from research experience with Autism Spectrum Disorder. Patient Educ Couns 101, 352361 (2018).

10. Geschwind, D.H. \& State, M.W. Gene hunting in autism spectrum disorder: on the path to precision medicine. Lancet Neurol 14, 1109-20 (2015).

11. Vorstman, J.A.S. et al. Autism genetics: opportunities and challenges for clinical translation. Nat Rev Genet 18, 362-376 (2017).

12. American_Psychiatric_Association \& DSM_Task_Force. Diagnostic and statistical manual of mental disorders : DSM-5. (2013).

13. Lord, C. \& Bishop, S.L. Recent advances in autism research as reflected in DSM-5 criteria for autism spectrum disorder. Annu Rev Clin Psychol 11, 53-70 (2015).

This review of advances in scientific knowledge about ASD uses DSM-5 diagnostic criteria as a framework for the discussion.

14. Sanders, S.J. Next-Generation Sequencing in Autism Spectrum Disorder. Cold Spring Harb Perspect Med 9(2019).

15. Ganna, A. et al. Quantifying the Impact of Rare and Ultra-rare Coding Variation across the Phenotypic Spectrum. Am J Hum Genet 102, 1204-1211 (2018).

16. He, Z., Xu, B., Buxbaum, J. \& Ionita-Laza, I. A genome-wide scan statistic framework for whole-genome sequence data analysis. Nat Commun 10, 3018 (2019).

17. Werling, D.M. et al. An analytical framework for whole-genome sequence association studies and its implications for autism spectrum disorder. Nat Genet 50, 727-736 (2018).

18. Hoang, N., Buchanan, J.A. \& Scherer, S.W. Heterogeneity in clinical sequencing tests marketed for autism spectrum disorders. NPJ Genom Med 3, 27 (2018).

This paper presents a comprehensive survey of sequencing tests for ASD that are primarily being marketed by commercial laboratories as adjuncts or follow-up to chromosomal microarrays.

19. Strande, N.T. et al. Evaluating the Clinical Validity of Gene-Disease Associations: An Evidence-Based Framework Developed by the Clinical Genome Resource. Am J Hum Genet 100, 895-906 (2017).

This work presents the latest ClinGen classifications and framework recommendations to assess the strength of gene-disease relationships.

20. Smith, E.D. et al. Classification of Genes: Standardized Clinical Validity Assessment of Gene-Disease Associations Aids Diagnostic Exome Analysis and Reclassifications. Hum Mutat 38, 600-608 (2017).

21. Rehm, H.L. et al. ClinGen--the Clinical Genome Resource. N Engl J Med 372, 2235-42 (2015).

22. Rivera-Munoz, E.A. et al. ClinGen Variant Curation Expert Panel experiences and standardized processes for disease and gene-level specification of the ACMG/AMP guidelines for sequence variant interpretation. Hum Mutat 39, 1614-1622 (2018).

23. Angione, K., Gibbons, M. \& Demarest, S. An Objective Method for Evaluating Next-Generation Sequencing Panels. J Child Neurol, 883073818815036 (2018).

24. Pitini, E. et al. How is genetic testing evaluated? A systematic review of the literature. Eur J Hum Genet 26, 605-615 (2018).

25. Sahin, M. \& Sur, M. Genes, circuits, and precision therapies for autism and related neurodevelopmental disorders. Science 350(2015).

26. Bale, T.L. et al. Early life programming and neurodevelopmental disorders. Biol Psychiatry 68, 314-9 (2010). 
27. Gray, S.J. Gene therapy and neurodevelopmental disorders. Neuropharmacology 68, 136-42 (2013).

28. Gonzalez-Mantilla, A.J., Moreno-De-Luca, A., Ledbetter, D.H. \& Martin, C.L. A Cross-Disorder Method to Identify Novel Candidate Genes for Developmental Brain Disorders. JAMA Psychiatry 73, 275-83 (2016).

29. Moreno-De-Luca, A. et al. Developmental brain dysfunction: revival and expansion of old concepts based on new genetic evidence. Lancet Neurol 12, 406-14 (2013).

This conceptual paper hypothesizes for the many ASD-relevant genes currently described as variably penetrant that, when the disorders encompassed by developmental brain dysfunction are considered as a group, the penetrance of these genes may approach $100 \%$.

30. Srivastava, S. et al. Meta-analysis and multidisciplinary consensus statement: exome sequencing is a firsttier clinical diagnostic test for individuals with neurodevelopmental disorders. Genet Med (2019).

This scoping review and meta-analysis leads to recommendations that exome sequencing should become a first-tier diagnostic test for NDDs, including ASD.

31. Carter, M.T. \& Scherer, S.W. Autism spectrum disorder in the genetics clinic: a review. Clinical Genetics 83, 399-407 (2013).

32. Yuen, R.K. et al. Whole-genome sequencing of quartet families with autism spectrum disorder. Nat Med 21, 185-91 (2015).

This study finds that affected siblings can carry different ASD-relevant mutations, and when they do, they tend to demonstrate more clinical variability than those who share a variant.

33. Yuen, R.K. et al. Whole genome sequencing resource identifies 18 new candidate genes for autism spectrum disorder. Nat Neurosci (2017).

Using genome sequence and comprehensive annotation, this study finds that participants bearing mutations in ASD-relevant genes have lower adaptive ability than those who do not.

34. Satterstrom, F.K. et al. Large-Scale Exome Sequencing Study Implicates Both Developmental and Functional Changes in the Neurobiology of Autism. Cell 180, 568-584 e23 (2020).

This large-scale exome sequencing study shows many ASD-relevant genes as conferring risk for ASD or for ASD with neurodevelopmental delay, based on whether a gene has a higher frequency of disruptive de novo variants in ASD or neurodevelopmental delay.

35. Matson, J.L. \& Shoemaker, M. Intellectual disability and its relationship to autism spectrum disorders. Res Dev Disabil 30, 1107-14 (2009).

36. Skuse, D.H. Rethinking the nature of genetic vulnerability to autistic spectrum disorders. Trends Genet 23, 387-95 (2007).

This important discussion of the relationship between ASD and ID includes the idea that the presence of both characteristics in an individual increases the clinical ascertainment.

37. Shattuck, P.T. The contribution of diagnostic substitution to the growing administrative prevalence of autism in US special education. Pediatrics 117, 1028-37 (2006).

38. Nevison, C.D. \& Blaxill, M. Diagnostic Substitution for Intellectual Disability: A Flawed Explanation for the Rise in Autism. J Autism Dev Disord 47, 2733-2742 (2017).

39. Croen, L.A., Grether, J.K., Hoogstrate, J. \& Selvin, S. The changing prevalence of autism in California. J Autism Dev Disord 32, 207-15 (2002).

40. Ingram, D.H., Mayes, S.D., Troxell, L.B. \& Calhoun, S.L. Assessing children with autism, mental retardation, and typical development using the Playground Observation Checklist. Autism 11, 311-9 (2007).

41. Ventola, P. et al. Differentiating between autism spectrum disorders and other developmental disabilities in children who failed a screening instrument for ASD. J Autism Dev Disord 37, 425-36 (2007).

42. Pedersen, A.L. et al. DSM Criteria that Best Differentiate Intellectual Disability from Autism Spectrum Disorder. Child Psychiatry Hum Dev 48, 537-545 (2017).

43. Mooney, E.L., Gray, K.M. \& Tonge, B.J. Early features of autism: Repetitive behaviours in young children. Eur Child Adolesc Psychiatry 15, 12-8 (2006).

44. Osterling, J.A., Dawson, G. \& Munson, J.A. Early recognition of 1-year-old infants with autism spectrum disorder versus mental retardation. Dev Psychopathol 14, 239-51 (2002).

45. Baranek, G.T. Autism during infancy: a retrospective video analysis of sensory-motor and social behaviors at 9-12 months of age. J Autism Dev Disord 29, 213-24 (1999).

46. Clifford, S.M. \& Dissanayake, C. The early development of joint attention in infants with autistic disorder using home video observations and parental interview. J Autism Dev Disord 38, 791-805 (2008).

47. Mitchell, S., Cardy, J.O. \& Zwaigenbaum, L. Differentiating autism spectrum disorder from other developmental delays in the first two years of life. Dev Disabil Res Rev 17, 130-40 (2011).

48. Brereton, A.V., Tonge, B.J. \& Einfeld, S.L. Psychopathology in children and adolescents with autism compared to young people with intellectual disability. J Autism Dev Disord 36, 863-70 (2006). 
49. Barrett, B. et al. Comparing service use and costs among adolescents with autism spectrum disorders, special needs and typical development. Autism 19, 562-9 (2015).

50. Weitlauf, A.S. et al. in Therapies for Children With Autism Spectrum Disorder: Behavioral Interventions Update (Rockville (MD), 2014).

51. Helbig, I. et al. The ClinGen Epilepsy Gene Curation Expert Panel-Bridging the divide between clinical domain knowledge and formal gene curation criteria. Hum Mutat 39, 1476-1484 (2018).

52. World Health Organization. International statistical classification of diseases and related health problems, (World Health Organization, Geneva, 2004).

53. Fernandez, B.A. \& Scherer, S.W. Syndromic autism spectrum disorders: moving from a clinically defined to a molecularly defined approach. Dialogues Clin Neurosci 19, 353-371 (2017).

54. Leblond, C.S. et al. Meta-analysis of SHANK Mutations in Autism Spectrum Disorders: a gradient of severity in cognitive impairments. PLoS Genet 10, e1004580 (2014).

This study of the SHANK1, SHANK2 and SHANK3 genes in ASD demonstrates that mutations are detected in the entire spectrum of autism with a gradient of severity in cognitive impairment.

55. Niarchou, M. et al. Psychiatric disorders in children with $16 \mathrm{p} 11.2$ deletion and duplication. Transl Psychiatry 9, 8 (2019).

56. Weiss, L.A. et al. Association between microdeletion and microduplication at $16 \mathrm{p} 11.2$ and autism. $N$ Engl J Med 358, 667-75 (2008).

57. Vorstman, J.A. et al. The 22q11.2 deletion in children: high rate of autistic disorders and early onset of psychotic symptoms. J Am Acad Child Adolesc Psychiatry 45, 1104-13 (2006).

58. Fiksinski, A.M. et al. Understanding the pediatric psychiatric phenotype of $22 q 11.2$ deletion syndrome. Am J Med Genet A (2018).

59. Soorya, L. et al. Prospective investigation of autism and genotype-phenotype correlations in $22 q 13$ deletion syndrome and SHANK3 deficiency. Mol Autism 4, 18 (2013).

60. De Rubeis, S. et al. Synaptic, transcriptional and chromatin genes disrupted in autism. Nature 515, 209-15 (2014).

61. Iossifov, I. et al. The contribution of de novo coding mutations to autism spectrum disorder. Nature 515, 216-21 (2014).

62. Bernier, R. et al. Disruptive CHD8 mutations define a subtype of autism early in development. Cell 158, 263-276 (2014).

This next-generation sequencing study identifies disruptions in CHD8 to define a distinct ASD subtype and reveal comorbidities between brain development and enteric innervation.

63. Sanders, S.J. et al. A framework for the investigation of rare genetic disorders in neuropsychiatry. Nat Med 25, 1477-1487 (2019).

64. Frazier, T.W. et al. Molecular and phenotypic abnormalities in individuals with germline heterozygous PTEN mutations and autism. Mol Psychiatry 20, 1132-8 (2015).

65. Fernandez, B.A. et al. Phenotypic spectrum associated with de novo and inherited deletions and duplications at 16p11.2 in individuals ascertained for diagnosis of autism spectrum disorder. J Med Genet 47, 195-203 (2010).

66. D'Angelo, D. et al. Defining the Effect of the $16 \mathrm{p} 11.2$ Duplication on Cognition, Behavior, and Medical Comorbidities. JAMA Psychiatry 73, 20-30 (2016).

67. Ross, P.J. et al. Synaptic Dysfunction in Human Neurons With Autism-Associated Deletions in PTCHD1-AS. Biol Psychiatry (2019).

68. De Rubeis, S. \& Buxbaum, J.D. Genetics and genomics of autism spectrum disorder: embracing complexity. Hum Mol Genet 24, R24-31 (2015).

69. Lim, E.T. et al. Rates, distribution and implications of postzygotic mosaic mutations in autism spectrum disorder. Nat Neurosci 20, 1217-1224 (2017).

This exome sequencing study identifies that somatic mutations constitute a significant proportion of de novo mutations and can contribute importantly to ASD phenotypes.

70. Lai, M.C., Baron-Cohen, S. \& Buxbaum, J.D. Understanding autism in the light of sex/gender. Mol Autism 6, 24 (2015).

71. Paaby, A.B. \& Rockman, M.V. The many faces of pleiotropy. Trends Genet 29, 66-73 (2013).

72. Vorstman, J.A. \& Ophoff, R.A. Genetic causes of developmental disorders. Curr Opin Neurol 26, 128-36 (2013).

73. Depienne, C. et al. Mechanisms for variable expressivity of inherited SCN1A mutations causing Dravet syndrome. J Med Genet 47, 404-10 (2010). 
74. Bassett, A.S. et al. Rare Genome-Wide Copy Number Variation and Expression of Schizophrenia in 22q11.2 Deletion Syndrome. Am J Psychiatry, appiajp201716121417 (2017).

75. Pizzo, L. et al. Rare variants in the genetic background modulate cognitive and developmental phenotypes in individuals carrying disease-associated variants. Genet Med 21, 816-825 (2019).

76. An, J.Y. et al. Genome-wide de novo risk score implicates promoter variation in autism spectrum disorder. Science 362(2018).

77. Garg, P. \& Sharp, A.J. Screening for rare epigenetic variations in autism and schizophrenia. Hum Mutat (2019).

78. Andrews, S.V. et al. Case-control meta-analysis of blood DNA methylation and autism spectrum disorder. Mol Autism 9, 40 (2018).

79. Hagerman, R., Au, J. \& Hagerman, P. FMR1 premutation and full mutation molecular mechanisms related to autism. J Neurodev Disord 3, 211-24 (2011).

80. Modabbernia, A., Velthorst, E. \& Reichenberg, A. Environmental risk factors for autism: an evidence-based review of systematic reviews and meta-analyses. Mol Autism 8, 13 (2017).

81. Grove, J. et al. Identification of common genetic risk variants for autism spectrum disorder. Nat Genet 51, 431-444 (2019).

82. Coe, B.P. et al. Neurodevelopmental disease genes implicated by de novo mutation and copy number variation morbidity. Nat Genet 51, 106-116 (2019).

83. Jacquemont, S. et al. A higher mutational burden in females supports a "female protective model" in neurodevelopmental disorders. Am J Hum Genet 94, 415-25 (2014).

84. Larsen, E. et al. A systematic variant annotation approach for ranking genes associated with autism spectrum disorders. Mol Autism 7, 44 (2016).

85. Abrahams, B.S. et al. SFARI Gene 2.0: a community-driven knowledgebase for the autism spectrum disorders (ASDs). Mol Autism 4, 36 (2013).

86. Hyman, S.L., Levy, S.E., Myers, S.M., Council On Children With Disabilities, S.O.D. \& Behavioral, P. Identification, Evaluation, and Management of Children With Autism Spectrum Disorder. Pediatrics 145(2020).

87. lakoucheva, L.M., Muotri, A.R. \& Sebat, J. Getting to the Cores of Autism. Cell 178, 1287-1298 (2019).

88. Robinson, E.B., Lichtenstein, P., Anckarsater, H., Happe, F. \& Ronald, A. Examining and interpreting the female protective effect against autistic behavior. Proc Natl Acad Sci U S A 110, 5258-62 (2013).

89. Pinto, D. et al. Convergence of genes and cellular pathways dysregulated in autism spectrum disorders. Am J Hum Genet 94, 677-94 (2014).

90. Lionel, A.C. et al. Disruption of the ASTN2/TRIM32 locus at 9q33.1 is a risk factor in males for autism spectrum disorders, ADHD and other neurodevelopmental phenotypes. Hum Mol Genet 23, 2752-68 (2014).

91. Woodbury-Smith, M. et al. Variable phenotype expression in a family segregating microdeletions of the NRXN1 and MBD5 autism spectrum disorder susceptibility genes. NPJ Genom Med 2(2017).

92. Leppa, V.M. et al. Rare Inherited and De Novo CNVs Reveal Complex Contributions to ASD Risk in Multiplex Families. Am J Hum Genet 99, 540-554 (2016).

93. Yu, T.W. et al. Using whole-exome sequencing to identify inherited causes of autism. Neuron 77, 259-73 (2013).

94. Rejeb, I. et al. First case report of Cohen syndrome in the Tunisian population caused by VPS13B mutations. BMC Med Genet 18, 134 (2017).

95. Daoud, H. et al. Autism and nonsyndromic mental retardation associated with a de novo mutation in the NLGN4X gene promoter causing an increased expression level. Biol Psychiatry 66, 906-10 (2009).

96. Risi, S. et al. Combining information from multiple sources in the diagnosis of autism spectrum disorders. $J$ Am Acad Child Adolesc Psychiatry 45, 1094-1103 (2006).

97. Wang, T. et al. De novo genic mutations among a Chinese autism spectrum disorder cohort. Nat Commun 7, 13316 (2016).

98. Li, J. et al. Targeted sequencing and functional analysis reveal brain-size-related genes and their networks in autism spectrum disorders. Mol Psychiatry 22, 1282-1290 (2017).

99. Sato, D. et al. SHANK1 Deletions in Males with Autism Spectrum Disorder. American Journal of Human Genetics 90, 879-887 (2012).

100. Zhang, L. et al. A promoter variant in ZNF804A decreasing its expression increases the risk of autism spectrum disorder in the Han Chinese population. Transl Psychiatry 9, 31 (2019).

101. Gouder, L. et al. Altered spinogenesis in iPSC-derived cortical neurons from patients with autism carrying de novo SHANK3 mutations. Sci Rep 9, 94 (2019). 
102. Khalil, R. et al. PSMD12 haploinsufficiency in a neurodevelopmental disorder with autistic features. Am J Med Genet B Neuropsychiatr Genet 177, 736-745 (2018).

103. Pascolini, G. et al. Autism spectrum disorder in a patient with a genomic rearrangement that only involves the EPHA5 gene. Psychiatr Genet 29, 86-90 (2019).

104. O'Roak, B.J. et al. Sporadic autism exomes reveal a highly interconnected protein network of de novo mutations. Nature 485, 246-50 (2012).

105. Manning, M.A. et al. Terminal 22q deletion syndrome: a newly recognized cause of speech and language disability in the autism spectrum. Pediatrics 114, 451-457 (2004).

106. Merner, N. et al. A de novo frameshift mutation in chromodomain helicase DNA-binding domain 8 (CHD8): A case report and literature review. Am J Med Genet A 170, 1225-35 (2016).

107. Imaizumi, T., Kumakura, A., Yamamoto-Shimojima, K., Ondo, Y. \& Yamamoto, T. Identification of a rare homozygous SZT2 variant due to uniparental disomy in a patient with a neurodevelopmental disorder. Intractable Rare Dis Res 7, 245-250 (2018). 
Table 1. Aspects of ASD that may affect gene curation

\begin{tabular}{|ll|}
\hline ASD consideration & Issue \\
\hline Phenotype & \\
\hline $\begin{array}{l}\text { Diagnostic process in ASD varies with differences in } \\
\text { the quality of the assessment }\end{array}$ & Is the phenotype ASD? \\
\hline $\begin{array}{l}\text { Sex of the affected individual and the parent } \\
\text { transmitting the variant will impact expression of ASD } \\
\text { phenotype for some genes }\end{array}$ & $\begin{array}{l}\text { How will sex of the individuals or transmitting } \\
\text { parents be factored into evaluation of association } \\
\text { evidence? }\end{array}$ \\
\hline Genotype & $\begin{array}{l}\text { How can function/alterations be assessed if } \\
\text { disease mechanism is unclear? }\end{array}$ \\
\hline $\begin{array}{l}\text { The etiopathology of ASD is only partly known and } \\
\text { may converge on different pathways }\end{array}$ & $\begin{array}{l}\text { What weight to assign inherited vs. de novo } \\
\text { variants? }\end{array}$ \\
\hline $\begin{array}{l}\text { Unlike most disorders, ASD and ASD traits may be } \\
\text { present in the parents and/or siblings }\end{array}$ & $\begin{array}{l}\text { How does scoring system account for lower impact } \\
\text { genes? }\end{array}$ \\
\hline $\begin{array}{l}\text { Methodological } \\
\text { databases, but more prevalent in ASD cohorts }\end{array}$ & $\begin{array}{l}\text { How to evaluate results from large case-control } \\
\text { data? }\end{array}$ \\
\hline $\begin{array}{l}\text { Case-control studies for ASD do not typically match } \\
\text { for age, ethnicity, and sex }\end{array}$ & $\begin{array}{l}\text { Is the observed animal behaviour reflective of ASD } \\
\text { or something else? }\end{array}$ \\
\hline $\begin{array}{l}\text { The interpretation of behavioural findings from } \\
\text { animal models for ASD is challenging }\end{array}$ & \\
\hline
\end{tabular}

Table 2. Scoring outcome in 11 exemplary genes

\begin{tabular}{|l|l|l|}
\hline Gene & Existing ClinGen Curation & $\begin{array}{l}\text { Proposed additional annotation of evidence in support } \\
\text { of a relationship with ASD }\end{array}$ \\
\hline$A D N P$ & NA & Definitive (score $=14.5$ ) \\
\hline ANK2 & Definitive (Brugada Syndrome) & Moderate (score $=10.8$ ) \\
\hline$A R I D 1 B$ & Definitive (Coffin-Siris Syndrome) & Definitive (score $=12$ ) \\
\hline DSCAM & NA & Definitive (score $=12$ ) \\
\hline KATNAL2 & NA & Limited (score $=4.1$ ) \\
\hline KDM5B & NA & Limited (score $=2.8$ ) \\
\hline NRXN2 & NA & Moderate (score $=7$ ) \\
\hline NLGN4X & Definitive (Complex NDD) & Definitive (score $=12$ ) \\
\hline MEF2C & Definitive (Complex NDD) & Moderate (score $=9.85$ ) \\
\hline SYN1 & Moderate (Complex NDD) & Limited (score $=2.35$ ) \\
\hline VPS13B & Definitive (Cohen Syndrome) & Limited (score $=6.35$ ) \\
\hline
\end{tabular}

See Supplementary Table 1 for a detailed breakdown of the scoring of each gene. ASD, autism spectrum disorder; ClinGen, Clinical Genome; NA, not available; NDD, neurodevelopmental disorder. ${ }^{a}$ Calculated classification as outlined in the ClinGen protocol (https://clinicalgenome.org; and see SOPVersion 6, August 29, 2018). 


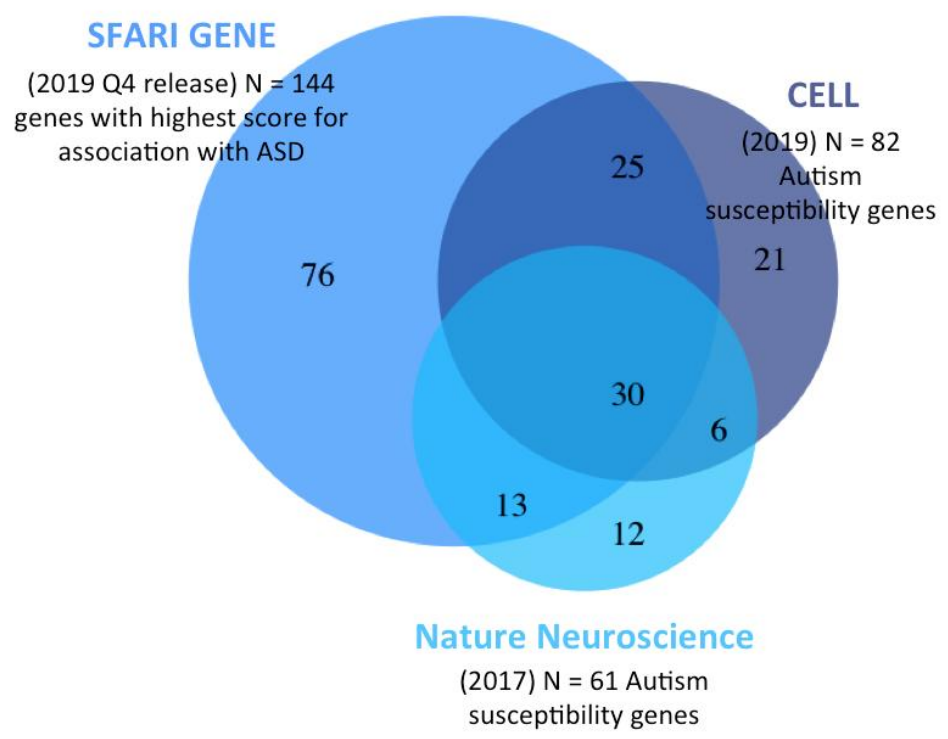

Fig. 1. Overlap between three sets of genes considered to be associated with ASD susceptibility. At the time of the submission of the manuscript, SFARI GENE (https://gene.sfari.org) has scored 913 genes; of these, 144 genes received the highest score (Category 1; High Confidence). lakoucheva et al. ${ }^{87}$ reported 106 ASD susceptibility genes, of which 82 have been evaluated by SFARI Gene to date. Yuen et al. ${ }^{33}$ reported 61 ASD susceptibility genes, all which have been evaluated by SFARI Gene to date. In both gene sets ${ }^{33,87}$ a third of genes did not yield the highest SFARI score for ASD association, and only a total of 30 genes were shared between these three gene sets. 


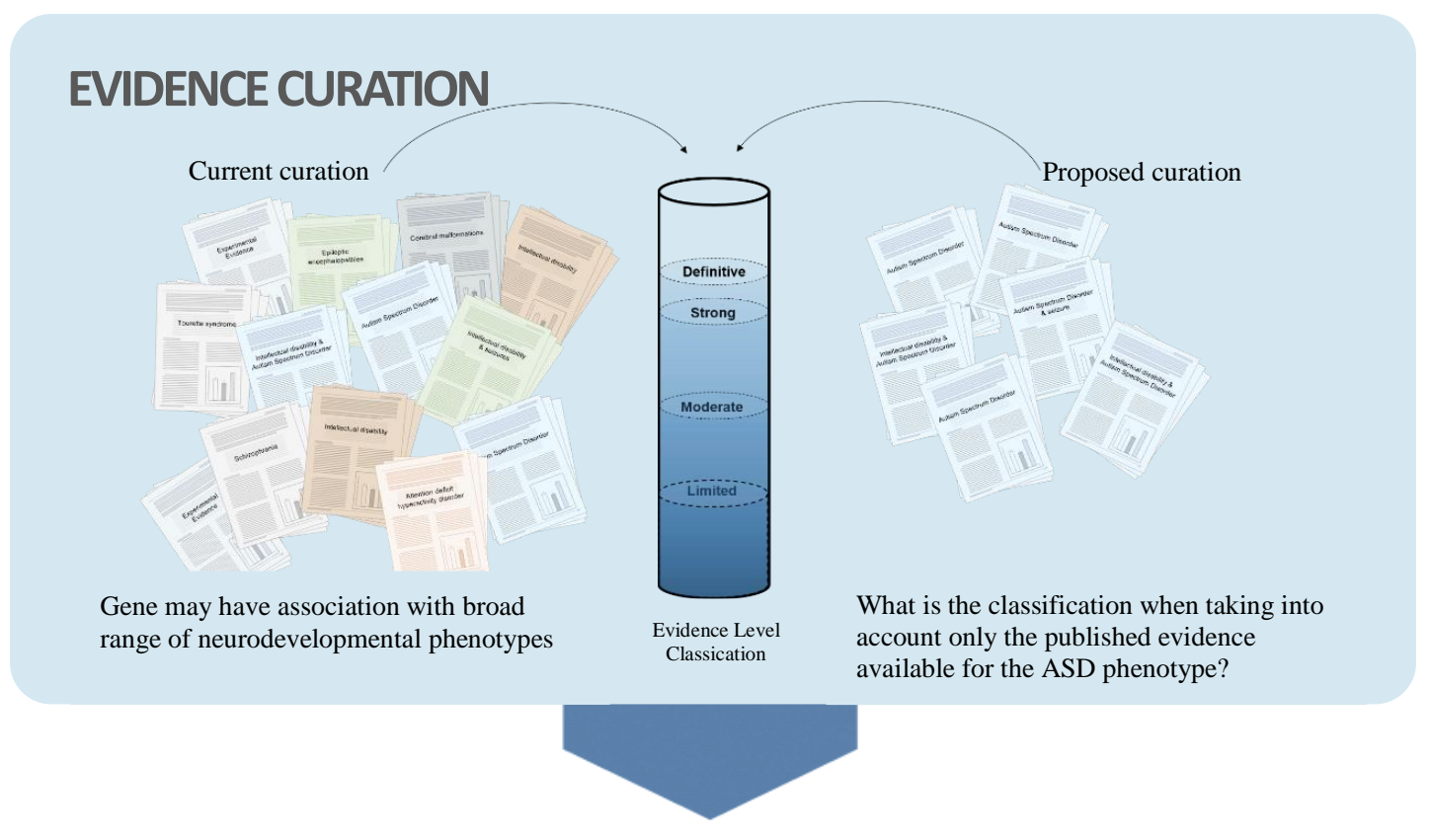

\section{PHENOTYPE}

- Contextualize ASD

phenotype with intellectual

ability data.

-What is the level of

confidence in ASD

phenotype?

\section{GENOTYPE}

\section{ClinGen curation procedure with quality rating scale for the report of ASD phenotype}

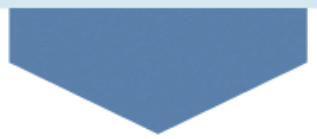

\section{LIST}

- Compilation of genes relevant to ASD

\section{NDD genes with strong/definitive evidence for association with ASD}

Fig. 2. Proposed pipeline. Step 1, Evidence curation to evaluate strength of a gene-disease relationship based on publicly available genetic and experimental evidence. Select gene with reported association with ASD, regardless of additional phenotypic associations. We will start with genes with highest level of available evidence. Step 2, PHENOTYPE and GENOTYPE data: consists of two components, i) systematic evaluation and consensus rating of the quality of report of the ASD phenotypes in the evidence collected by the ClinGen procedure (Table 1), and ii) application of the ClinGen evaluation process for genetic evidence as outlined by Strande et al. Table 2). Step 3, LIST compilation: any NDD gene that scores as strong or definitive category for association with ASD will be included on the list. 
A. Expert clinician or multidisciplinary team assigned (consensus) diagnosis of ASD

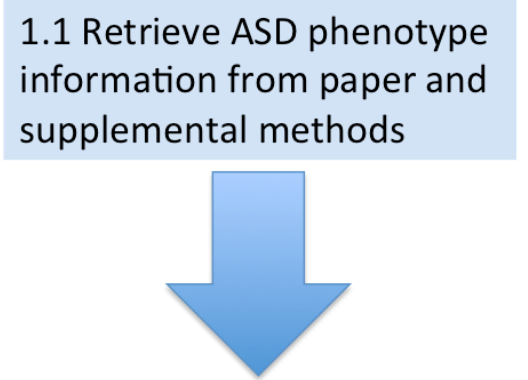

1.2 Retrieve information on intellectual ability

B. Validated assessment methods were used (e.g. ADI-R, ADOS)

C. Explicit mention of "[meeting] DSM or ICD [criteria]"

D. Description of symptoms in social/communicative AND repetitive domain indicative of a diagnosis of ASD

E. Only mentions "ASD", "Autism", "Autistic disorder", "PDD”, "PDD-NOS" or "Asperger"

F. Only mentions "Autistic features", "Autistic traits", "Autistic behavior" or "Autism-like behavior" (or similar terms with the same meaning) or only a description of some symptoms compatible with ASD but insufficient to qualify for $D$.

a. No ID, mild ID or moderate ID

b. Severe ID

c. Profound ID

d. No, or insufficient, information on intellectual ability.

\subsection{Rating ASD phenotype}

High confidence: $A, B, C$, or any combination of these.

Medium confidence: $D$ Low confidence: $\mathrm{E}$ or $\mathrm{F}$

\subsection{Cognitive ability cautionary comment}

If a: no cautionary comment required

If $b$, with A AND B: no cautionary comment required

If $b$, without A AND B: add comment "some uncertainty regarding validity of ASD diagnosis in light of severe ID and insufficient information on ASD phenotyping methods"

If c: add comment "case not counted towards evidence in light of profound ID" If $\mathrm{d}$ : "uncertainty regarding validity of ASD diagnosis in light of insufficient information regarding intellectual ability"

Fig 3. Systematic evaluation of quality of the ASD phenotype report. Six phenotype experts independently scored eight published reports ${ }^{100-107}$ using this algorithm; results indicated $98 \%$ consistency regarding the rating of low, medium and high confidence in the reported ASD phenotype, and $90 \%$ consistency regarding the cognitive ability information. Proposed scoring adjustments: If default score is 2 (ClinGen default for de novo variant): low confidence $(-1)$ and medium confidence $(-0.5)$; if default score is 1.5 (ClinGen default for an inherited variant that is predicted/proven null): low confidence (-0.5) and medium confidence $(-0.25)$; if default score is 0.5 (ClinGen default for an inherited variant not predicted/proven null, with some evidence of gene impact, e.g. missense variant with functional evidence supporting pathogenicity): low confidence (-0.25) and medium confidence (-0.1). For all default scores: Cognitive ability comments applied to all default scores: profound ID (c): case not counted towards curation; insufficient information regarding intellectual ability (d): scoring adjustments optional and decided by expert review. Note that these rules are tentative and may be adjusted based on increasing curation experience, but have been maintained consistently across all curations thus far. ADI-R, Autism Diagnostic Interview- Revised; ADOS, Autism Diagnostic Observation Schedule; DSM, Diagnostic and Statistical Manual of Mental Disorders; ICD, International Statistical Classification of Diseases; PDD, pervasive developmental disorder; PDD-NOS, pervasive developmental disorder-not otherwise specified. 
Box 1. Characteristics of the proposed list of genes relevant for ASD

This list will:

- Identify genes that have sufficient evidence of relevance to ASD when affected by deleterious genetic variants

- Provide a route towards identifying an underlying genetic condition that contributes to the ASD phenotype

- Contribute to insight into biological mechanisms involved in the etiopathology of ASD

This list will not:

- Replace the behavioural diagnosis

- Imply specificity for ASD alone (effects may be pleiotropic)

- Negate the need for an NDD, ID, or any other gene list

- Be fully comprehensive; rather, it is a work-in-progress and thus not to be used to exclude a clinical genetic diagnosis

- Predict specific type or severity of ASD

\section{Box 2. Guidelines for reporting ASD genotype-phenotype studies}

To maximize the informative potential of genetic studies in ASD, the following elements should be considered.

\section{Genetic information}

- Follow latest recommendations for sequence variant nomenclature.

- Report genome build and gene transcript in which variants are reported.

- Mention other variants found in addition to the primary one.

- Use p. and c. nomenclature of variant (where ' $p$ ' denotes protein reference sequence and ' $c$ ' denotes a coding DNA reference sequence); specify exact breakpoints of structural variants or note when unknown.

- Report technology that was employed to detect variant and whether findings were validated.

- Report the inheritance of the variant (de novo versus inherited) and possible segregation within the family.

- Report frequency in general population/control databases at time of publication

\section{Phenotype information}

- Describe how the diagnosis of ASD was made; the involved clinical expertise and what (validated) ASD assessment tools were employed.

- Report any available information on intellectual ability, including the test(s) administered and scores.

- If formal intellectual assessment was performed, use adequate classification terms in accordance with Diagnostic and Statistical Manual of Mental Disorders and International statistical Classification of Diseases criteria.

- Large cohort studies should include a (supplementary) table with the above-mentioned information for each subject, or at minimum for those subjects identified with (putatively) deleterious genetic variants. Tables should be organized such that phenotype and genotype information can be evaluated together.

The highest degree of information can be obtained when parental phenotypic information is provided, recognizing that features of ASD can be present (or present at sub-clinical levels). If parents are reported as without any ASD or related difficulties, investigators should report whether parents were formally assessed, and if so, what instruments were used. 